\title{
Control of the Lateral Organization in Langmuir Monolayers via Molecular Aggregation of Dyes
}

\author{
Antonio M. González-Delgado, ${ }^{\dagger}$ Carlos Rubia-Payá, ${ }^{\dagger}$ Cristina Roldán-Carmona, ${ }^{\dagger}$ \\ Juan J. Giner-Casares, ${ }^{\dagger, \dagger}$ Marta Pérez-Morales, ${ }^{\dagger}$ Eulogia Muñoz, ${ }^{\dagger}$ María T. Martín-Romero, ${ }^{\dagger}$ \\ Luis Camacho, ${ }^{*, \dagger}$ and Gerald Brezesinski ${ }^{*}$
}

Department of Physical Chemistry and Applied Thermodynamics, University of Córdoba, Campus de Rabanales, Edificio Marie Curie, Córdoba, Spain E-14014, and Interfaces Department, Max Planck Institute of Colloids and Interfaces, Science Park Golm, 14476 Potsdam, Germany

Received: August 13, 2010

\begin{abstract}
This paper shows that it is possible to construct well-defined 2D structures at the air-water interface in which the lateral organization is controlled by means of the preparation of mixed films, and selecting the components so that there are attractive interactions between them. The goal here is to establish the lateral connection between components through self-aggregation of the dye. This can be achieved by selecting a suitable balance between the sizes of the hydrophobic and polar groups. In such a way, the domain structure depends on the ability of the tilt dye to fill the available area. Thus, the molecular organization and the domain morphology of mixed films containing dimyristoyl-phosphatidic acid (DMPA) and the hemicyanine dye, 4-[4-(dimethylamino)styryl]-1-docosylpyridinium bromide (SP), have been studied by using Grazing Incidence X-ray Diffraction (GIXD), Brewster angle microscopy (BAM), and reflection spectroscopy at the air-water interface. For this mixed system, the formation of circular domains with bright horizontal regions and dark vertical regions was observed. Furthermore, depending on the temperature, it is observed as branches grow from circular domains, whose brightness depends on the growth direction. Thus, BAM images allow us to observe some branches that, as their growth direction changes, their brightness also changes simultaneously. The GIXD experiment permits us to relate the circular domains with an orthorhombic phase and the branches grown from the circles with an Overbeck phase. In both cases, the formed structures are induced by the hemicyanine aggregation. Circular BAM domain textures have been simulated by using the Fresnel equations for biaxial anisotropic materials.
\end{abstract}

\section{Introduction}

The development of methods to build well-ordered assemblies of molecules into larger structures is a current objective of supramolecular chemistry. ${ }^{1,2}$ In this sense, the air-water interface is an ideal model for these purposes, as it is easy to prepare in a pure state and also because the surface coverage can be smoothly adjusted by using the Langmuir trough technique. ${ }^{3}$ However, Langmuir monolayers are interesting not only in a fundamental way but also as a means to build an ordered system on mesoscopic length scales. ${ }^{4}$ The domains observed in Langmuir monolayers at high surface pressures indicate the formation of large structures. At the nanoscopic scale, the physical properties of these domains are determined by the relations between chemical composition, structure, and organization of these materials. ${ }^{5}$ However, at the mesoscopic scale the domain shape is controlled by the competition between the dipolar energy of the domain and the line tension energy. ${ }^{6}$ Thus, the repulsive dipolar interaction of the molecules within a domain favors a large boundary-to-area ratio, by which the electrostatic energy is reduced. ${ }^{7}$ In this case, the domain morphology does not entirely correspond to the molecular lattice structure. $^{8}$

\footnotetext{
* To whom correspondence should be addressed. E-mail: 1camacho@ uco.es.

University of Córdoba.

${ }^{\ddagger}$ Max Planck Institute of Colloids and Interfaces.
}

To design well-defined structures in which the lateral organization is controlled, we need somehow to compensate the repulsion energy between dipoles. One method to compensate this repulsion energy is to form monolayers containing different components with attractive interactions between them. Thus, Vollhardt et al. studied melamine-type monolayers, where the amphiphilic molecules are connected by hydrogen bonds through nonsurface-active species such as barbituric acid. ${ }^{2}$ This type of bonding leads to the formation of large domains with long-range orientation of the molecules. Another method to compensate the repulsion energy between dipoles, and used recently by us, ${ }^{8}$ is the preparation of mixed monolayers of an anionic phospholipid (DMPA) and a water-soluble cationic dye (methylene blue, MB), which is retained at the interface by electrostatic interactions, establishing lateral connections through self-aggregation of the dye.

This paper attempts to show that the method used in the DMPA:MB system can be applied in a general manner to other dyes that show self-aggregation, under the condition of a suitable balance between the sizes of the hydrophobic and polar groups. Thus, if $a_{\mathrm{c}}$ is the interfacial area occupied by the hydrophobic group when alkyl chains are fully extended, and $a_{0}$ is the minimum interfacial area occupied by the headgroup, dyes to be selected should obey $a_{\mathrm{c}} \geq a_{0}$. In this way, the domain structure depends on the ability of the dye to fill the available area excess $\left(a_{\mathrm{c}}-a_{0}\right)$. 
SCHEME 1: (A) Molecular Structures of Hemicyanine, SP, and Dimyristoyl-Phosphatidic Acid, DMPA and (B) Graphical Definition of the Transition Dipolar Moment, $\mu$, the Polar Tilt Angle, $\theta$, and the Azimuthal Angle, $\phi$, of the SP Molecules in the Presence of the DMPA Molecule (the interface plane coincides with the $x-y$ plane)

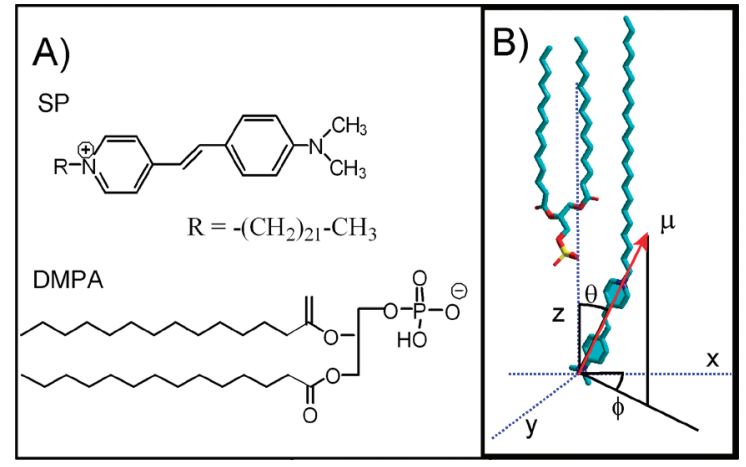

To verify the above approach, mixed films containing dimyristoylphosphatidic acid (DMPA) and an amphiphilic hemicyanine dye (SP) in a molar ratio of 1:1 have been studied. It is known that the hemicyanine group suffers $\mathrm{H}$-aggregation at the air-water interface, ${ }^{9-15}$ and therefore it is a suitable compound for the study indicated. The behavior of this mixed monolayer has been studied by using reflection spectroscopy, ${ }^{16-19}$ Brewster angle microscopy (BAM), ${ }^{20-25}$ as well as Grazing Incidence X-ray Diffraction (GIXD) ${ }^{5,26-30}$ The BAM technique has revealed an exciting large variety of morphological textures, also designated as condensed phase domains. These domains can exhibit a striking inner anisotropy and form subdomains of different brightness which have been attributed to regions of different molecular orientation. ${ }^{24,25,31-35}$ Specifically for the SP: DMPA $=1: 1$ system, we observed the formation of circular domains with bright horizontal regions and dark vertical regions. Furthermore, depending on the temperature, it is observed as branches grow from circular domains that brightness depends on the growth direction. Thus, BAM images allow us to see some branches that when changing their growth direction, their brightness changes simultaneously. In our system, the SP molecules absorb at $532 \mathrm{~nm}$ (wavelength of the laser used in BAM), being the SP absorption the origin of the appearance of the domain textures. The experimental circular domains textures have been simulated on the basis of the Fresnel equations for biaxial anisotropic materials on isotropic substrates. This simulation permits us to obtain information about the rearrangement of the dye polar group in the domains. Furthermore, additional information on the rearrangement of the alkyl chain has been obtained from GIXD.

\section{Experimental Section}

Materials. Hemicyanine dye, 4-[4-(dimethylamino)styryl]1-docosylpyridinium bromide (SP), and dimyristoylphosphatidic acid (DMPA) were purchased from Sigma-Aldrich and used as received. Their molecular structures are depicted in Scheme 1. A mixture of chloroform:methanol, ratio 3:1 (v/v), was used as the spreading solvent for solving both components. The pure solvents were obtained without purification from Aldrich. Ultrapure water, produced by a Millipore Milli-Q unit, pretreated by a Millipore reverse osmosis system $(>18.2 \mathrm{M} \Omega \mathrm{cm})$, was used as a subphase. The subphase temperature was $21^{\circ} \mathrm{C}$ with pH 5.7.

Methods. Two different models of Nima troughs (Nima Technology, Coventry, England) were used in this work, both provided with a Wilhelmy-type dynamometric system using a strip of filter paper: a NIMA 611D with one moving barrier for the measurement of the reflection spectra, and a NIMA 601, equipped with two symmetrical barriers to record BAM images. The monolayers were compressed at a speed of $\sim 0.1 \mathrm{~nm}^{2} \min ^{-1}$ molecule ${ }^{-1}$.

UV-visible reflection spectra at normal incidence as the difference in reflectivity $(\Delta R)$ of the dye film-covered water surface and the bare surface ${ }^{36}$ were obtained with a Nanofilm Surface Analysis Spectrometer (Ref SPEC, ${ }^{2}$ supplied by Accurion GmbH, Göttingen, Germany).

Images of the film morphology were obtained by Brewster angle microscopy (BAM) with a I-Elli2000 (Accurion GmbH), using a Nd:YAG diode laser with wavelength $532 \mathrm{~nm}$ and $50 \mathrm{~mW}$, which can be recorded with a lateral resolution of 2 $\mu \mathrm{m}$. The image processing procedure included a geometrical correction of the image, as well as a filtering operation to reduce interference fringes and noise. The microscope and the film balance were located on a table with vibration isolation (antivibration system MOD-2 S, Accurion, Göttingen, Germany) in a large class 100 clean room.

Grazing Incidence X-ray Diffraction measurements of the monolayer were performed at $21{ }^{\circ} \mathrm{C}$ at the BW1 beamline, HASYLAB, DESY (Hamburg, Germany). A Langmuir film balance equipped with a single movable barrier and a Wilhelmy plate for monitoring the lateral pressure was placed in a hermetically closed container filled with helium. At BW1, a monochromatic synchrotron X-ray beam $(\lambda=1.304 \AA)$ was adjusted to strike the helium/water interface at a grazing incidence angle $\alpha_{i}=0.85 \alpha_{c}\left(\alpha_{c}=0.13^{\circ}\right)$ and illuminated roughly $2 \times 50 \mathrm{~mm}^{2}$ of the surface. During the measurements, the trough was laterally moved to avoid any sample damage by the strong X-ray beam. A linear position-sensitive detector (PSD, MYTHEN, Switzerland) was rotated to scan the in-plane $Q_{x y}$ component values of the scattering vector. The vertical channels of the PSD measured the out-of-plane $Q_{z}$ component of the scattering vector between 0 and $0.8 \AA^{-1}$. The diffraction data consisted of Bragg peaks at diagnostic $Q_{x y}$ values. The accumulated position-resolved counts were corrected for polarization, effective area, and Lorentz factor. Model peaks taken as Lorentzian in the in-plane direction and as Gaussian in the out-of-plane direction were least-squares fitted to the measured intensities. The diffracted intensity normal to the interface was integrated over the $Q_{x y}$ window of the diffraction peak to calculate the corresponding Bragg rod. The thickness of the monolayer was estimated from the fwhm of the Bragg rod using $\sim 0.9(2 \pi) / \mathrm{fwhm}\left(Q_{z}\right)$. Experimental details are described in the literature. ${ }^{5,26-30}$

\section{Results and Discussion}

1. Surface Pressure-Area Isotherms and Brewster Angle Microscopy (BAM). The surface pressure-area $(\pi-A)$ isotherms of pure SP have been published previously. ${ }^{9,37}$ The limit area per molecule of hemicyanine in pure dye film is close to $0.33 \mathrm{~nm}^{2} .{ }^{13}$ BAM images of SP monolayers reveal inhomogeneous films (see the Supporting Information), with the coexistence of dark regions surrounded by bright areas. This situation persists at high surface pressures, which is indicative of an irregular structure in the monolayer formed. $\pi-A$ isotherms of SP show the dependence on the counterion type and concentration used, $, 13-15,37$ which significantly affects the H-aggregation shown for this compound at the air-water interface. ${ }^{9-15}$ Presumably, such inhomogeneity of the SP monolayers is due to the imbalance between the perpendicular sections of the alkyl 
chain $\left(\sim 0.2 \mathrm{~nm}^{2}\right)$ and of the polar group $\left(\sim 0.33 \mathrm{~nm}^{2}\right)$, which prevents the formation of organized large-size structures at the interface.

As a strategy to promote a proper balance between the sizes of the hydrophobic and hydrophilic groups, we have studied mixed monolayers of SP and DMPA at different molar fractions, $x=n_{\mathrm{SP}} /\left(n_{\mathrm{SP}}+n_{\mathrm{DMPA}}\right)$. Thus, for a molar ratio $x=0.5$ (SP: DMPA $=1: 1$ monolayers), the DMPA molecule provides two aliphatic chains to the set, so the minimum perpendicular section of the 1:1 mixture would be around $0.6 \mathrm{~nm}^{2}$ per SP molecule. The anionic DMPA can also offset the positive charge of the SP molecule, which favors the complete miscibility of the mixture. Finally, it has to be highlighted that the alkyl chains of DMPA are shorter than that of SP. Therefore, it is expected the DMPA headgroup is next to the hemicyanine pyridyl group, although close to the hydrophobic region of the assembly, so that the DMPA polar group does not avoid the hemicyanine aggregation by the dye molecular tilt (see the idealized sketch shown in Scheme 1B). Consequently, the hemicyanine group would have freedom of movement, bending properly to allow its aggregation.

Monolayers of SP:DMPA $=1: 1(x=0.5)$ have been formed at the air-water interface by the cospreading method. Figure 1 (top left) shows its surface pressure-area $(\pi-A)$ isotherm at $21{ }^{\circ} \mathrm{C}$. As can be seen, the takeoff of the isotherm occurs at 2.2 $\mathrm{nm}^{2} / \mathrm{SP}$ molecule, and the isotherm shows an overshoot at 10 $\mathrm{mN} / \mathrm{m}$ and $1.54 \mathrm{~nm}^{2}$, approximately. After the overshoot, the surface pressure increases again with the decreasing area per molecule. At $\pi=50 \mathrm{mN} / \mathrm{m}$, the surface area is $A=0.6 \mathrm{~nm}^{2} /$ SP molecule, which corresponds to three totally vertical alkyl chains, that is, two per DMPA molecule and one per SP molecule.

Simultaneous to the isotherm recording, the morphology of the mixed monolayer at the air-water interface is directly observed by BAM. At low surface pressure only the gas phase is observed (Figure 1A). When the surface area decreases, the monolayer appears to be homogeneous (Figure 1B), indicating the complete miscibility of the components. At $\pi \approx 8 \mathrm{mN} / \mathrm{m}$, small circular domains appear (Figure 1C). These circular domains have inner textures with different brightness. Thus, the circle regions located above and below are darker than the environment, while the lateral regions of the circle are brighter. Before the overshoot, some branches grow from the circles. The brightness of these branches depends on the direction of their growth, that is, those growing in the horizontal direction are dark, while they are bright along the vertical direction, which is coincident with the laser incidence. After the overshoot, BAM allows us to observe some branches changing the direction of their growth together with their brightness (Figure 1D). Under further compression, the branches grow lengthwise, but not transversally (see panels E and F of Figure 1). Finally, at high surface pressure, the surface is completely covered with branch domains with different brightness, depending on their longitudinal direction (Figure 1G,H).

On the other hand, the temperature has a dramatic effect on the morphology of the domains. Figure 2 (top left) shows the $\pi-A$ isotherms at 17,21 , and $25^{\circ} \mathrm{C}$ for the SP:DMPA $=1: 1$ monolayer. Under these conditions, the takeoff of the isotherms decreases and the isotherm overshoot is shifted to higher surface pressures as the temperature increases. However, at high surface pressure the isotherm areas are independent of the temperature. Figure 2 also shows BAM images recorded at 25 and $17{ }^{\circ} \mathrm{C}$. For both temperatures circular domains with inner textures can be observed. At $25^{\circ} \mathrm{C}$, and as in the previous case, branches
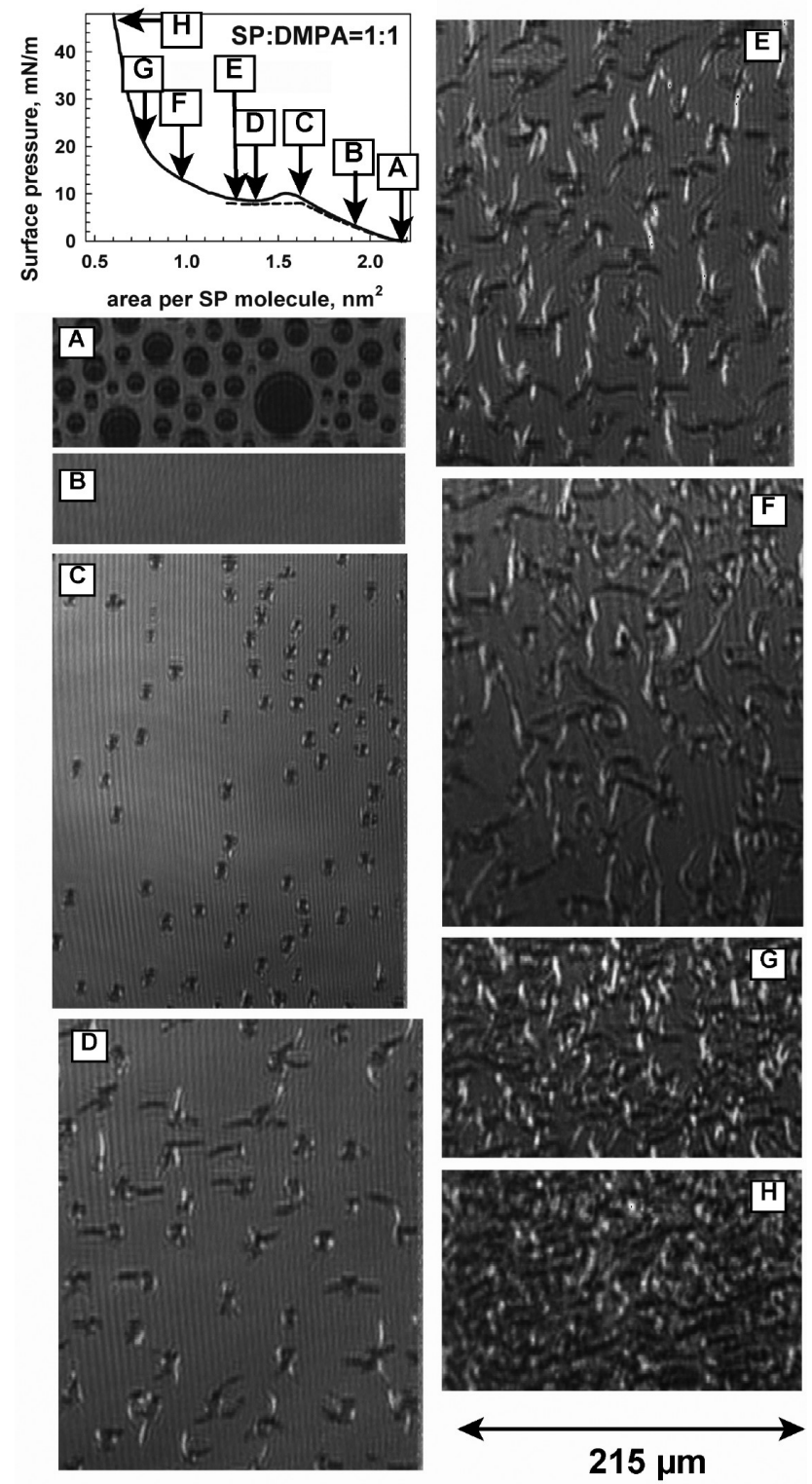

Figure 1. Surface pressure-area $(\pi-A)$ isotherm of the mixed SP: DMPA monolayer in a molar ratio of $1: 1$ at $T=21^{\circ} \mathrm{C}$ (top left). Dashed line represents the isotherm under compression from 0 to $8 \mathrm{mN} / \mathrm{m}$ and the area evolution at $8 \mathrm{mN} / \mathrm{m}$. $(\mathrm{A}-\mathrm{H}) \mathrm{BAM}$ images of the mixed SP: DMPA $=1: 1$ monolayer under different surface pressures at the air-water interface. Image size: $215 \mu \mathrm{m}$ width.

grow from these domains, which are wider and shorter on average than those observed at $21{ }^{\circ} \mathrm{C}$, and also the brightness of the branches depends on the direction of the growth (Figure 2B,C). Notoriously, there is not growth of branches at $17^{\circ} \mathrm{C}$ (Figure 2D-F). In any case, circular domains have the same inner textures as in the previous case.

The BAM images shown in panels D and E of Figure 2 correspond to the $\pi-A$ isotherm at $17{ }^{\circ} \mathrm{C}$, during the first compression cycle. When the monolayer is decompressed, and a second compression process is realized, some hysteresis at low surface pressure happens (see Figure 2, top left, gray line). The hysteresis at low surface pressure is related to the fact that the domains do not disappear completely when the monolayer is expanded. However, a significant phenomenon is observed during the second compression process, that is, the circular domains grow much more than those in the previous cases (see Figure $2 \mathrm{~F}, \mathrm{G}$ ). Initially (Figure $2 \mathrm{~F}$ ), circular domains have the 

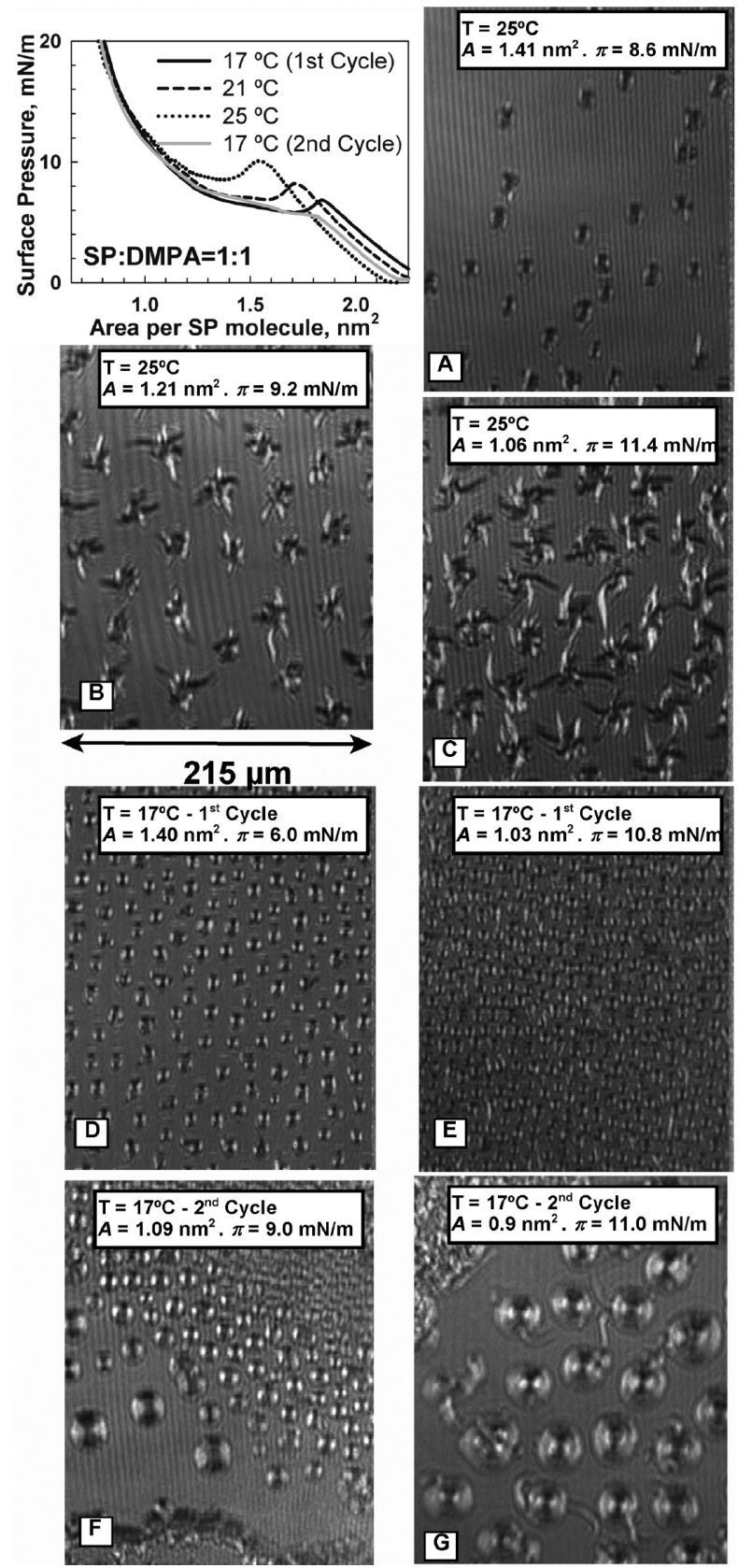

Figure 2. $\pi-A$ isotherms of the mixed SP:DMPA monolayer in a molar ratio of 1:1 at 17 (first and second compression processes), 21, and $25{ }^{\circ} \mathrm{C}$ (top right). (A-G) BAM images of the mixed SP:DMPA $=$ $1: 1$ monolayer under different surface pressures and temperatures. Image size: $215 \mu \mathrm{m}$ width.

same inner textures as those during the first compression. However, during the growth of such domains, the outer layers adopt a slightly different brightness, and result in the formation of branches (Figure 2G).

It is necessary to indicate that the isotherm overshoot cannot be observed during the second compression isotherm (Figure 3 , gray line), and neither for the $\pi-A$ curves obtained during the expansion of the monolayer (data not shown). An additional experiment was realized that consisted of comprising the SP: DMPA $=1: 1$ monolayer up to a surface pressure prior to the overshoot $(\pi \approx 8 \mathrm{mN} / \mathrm{m})$, and the spontaneous evolution of the monolayer at this constant pressure was registered (see top left in Figure 1, dashed line). We observed that the area decreased to a value close to $1.2 \mathrm{~nm}^{2} /$ per SP molecule, while BAM images showed circular domains and branches growing from the circles,
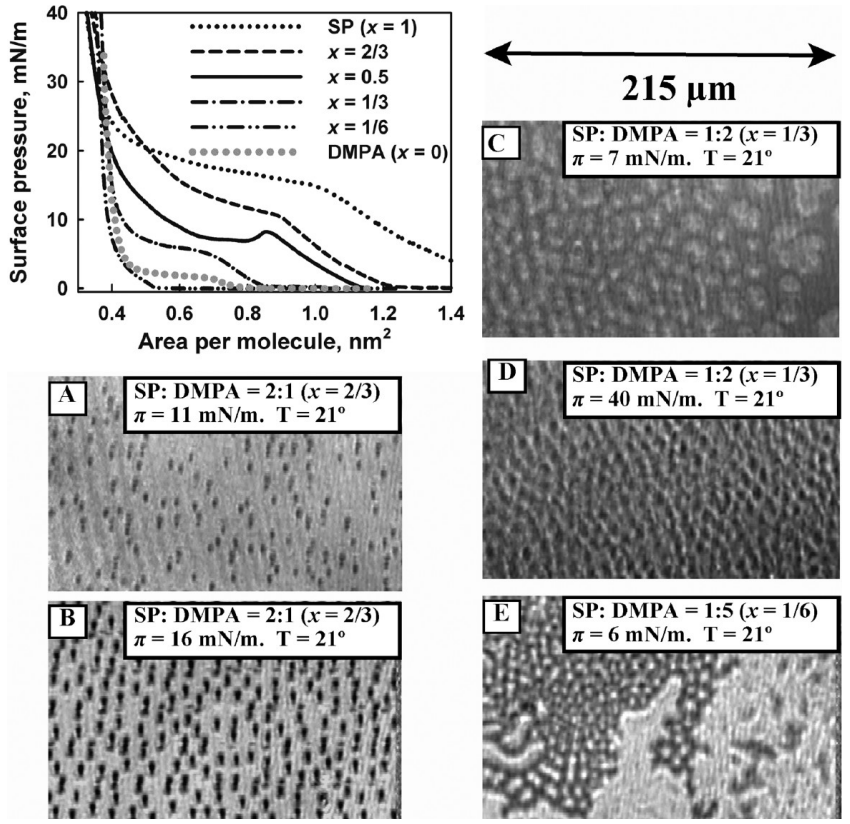

Figure 3. Surface pressure-area $(\pi-A)$ isotherms of the mixed SP: DMPA monolayer at various mole fractions of SP $(x)$ at $T=21{ }^{\circ} \mathrm{C}$. The isotherm areas are expressed per molecule (top left). (A-E) BAM images of the mixed SP:DMPA monolayer at different $x$ values. The experimental conditions are indicated in the figure. Image size: 215 $\mu \mathrm{m}$ width.

with similar textures as those described in Figure 1. On the basis of the above, the existence of the isotherm overshoot could be interpreted in terms of a dynamic jump, ${ }^{38}$ related to the kinetics of the monomer attachment to 2D aggregates. In fact, the formation of oligomers results in the decrease of the excess surface pressure of the monolayer. In these cases, the existence of the overshoot can be qualitatively explained by the fact that the aggregation rate is relatively slow due to the increase of the number of monomers in the aggregate.

It should be noted that the BAM images of pure DMPA monolayer or pure SP monolayer do not show the morphological behavior observed in Figures 1 and 2. ${ }^{39,40}$ It has been shown how some macrocations can be electrostatically attached to a DMPA matrix, ${ }^{41-46}$ forming domains without internal textures, because of weak or no aggregation between these macrocations. However, hexagonal and circular domains with inner textures were observed for mixed DMPA/Methylene Blue (MB) monolayers, ${ }^{8}$ although those circular domains had different textures in comparison with those observed here. As for MB, we can assert that the molecular aggregation of SP induces the formation of domains with well-organized structure. Moreover, the texture of the domains observed depending on their orientation is indicative of anisotropic structures. An additional fact that deserves mention is the absence of the coalescence between domains.

Figure 3 shows the $\pi-A$ isotherms of SP:DMPA monolayers, obtained at various molar fractions of SP, as well as some BAM images recorded simultaneously. In this figure, the isotherm areas are expressed per molecule. It has to be pointed out that the isotherm overshoot could not be observed for $x \neq 0.5$. For $x=2 / 3$, small circular domains with inner textures and without branch growth can be observed by BAM (Figure 3A,B). However, in this mixture the domains are poorly displayed, because the reflectivity of the brightest areas of the domains is similar to the reflectivity of the surrounding areas. For $x=1 / 3$ (Figure 3C,D), domains without inner textures are observed 

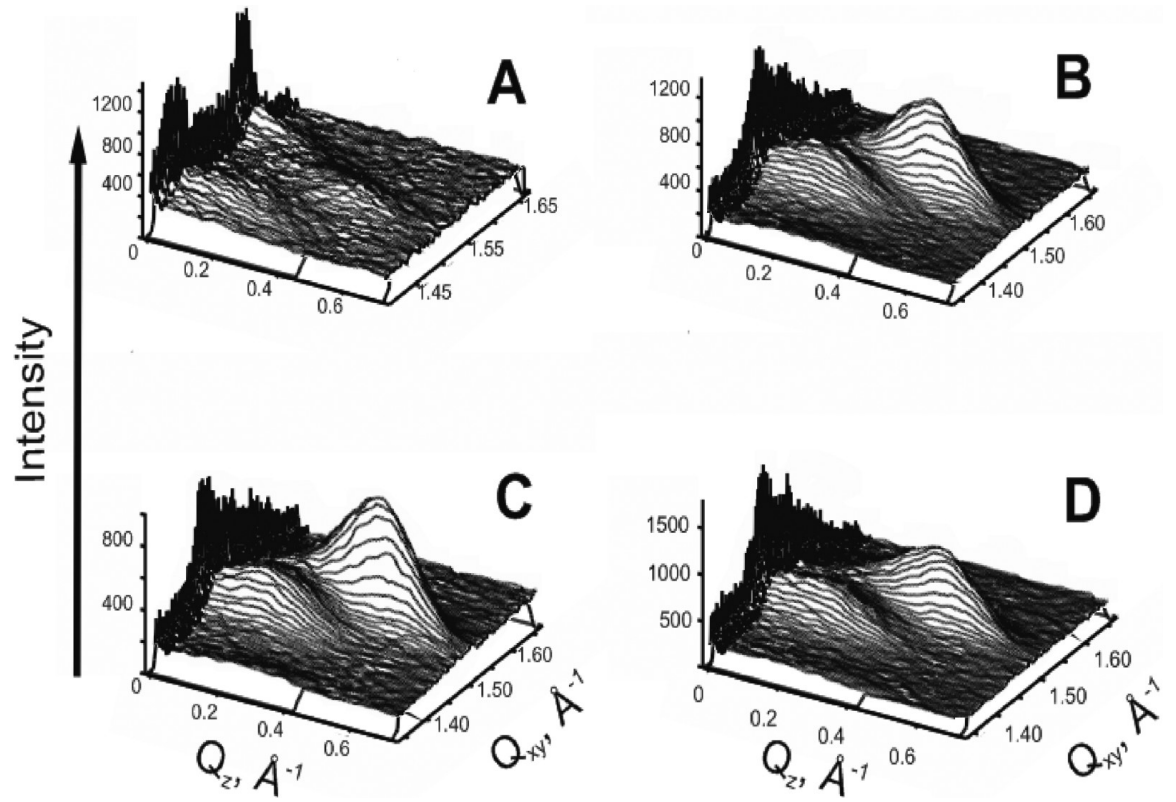

Figure 4. 3D plots of the corrected X-ray intensities versus the in-plane and out-of-plane scattering vector component $Q_{x y}$ and $Q_{z}$ for the mixed SP:DMPA monolayer in a molar ratio of $1: 1$ at $T=21^{\circ} \mathrm{C}$. (A) $\pi=10 \mathrm{mN} / \mathrm{m}$, before surface pressure overshoot (see isotherm of Figure 1). (B) $\pi=10 \mathrm{mN} / \mathrm{m}$, after ca. $20 \mathrm{~min}$ of maintaining the surface pressure constant (after the overshoot). (C) $\pi=10 \mathrm{mN} / \mathrm{m}$, after ca. $40 \mathrm{~min}$ of maintaining the surface pressure constant. (D) $\pi=25 \mathrm{mN} / \mathrm{m}$.

TABLE 1: In-Plane $Q_{x y}$ and Out-of-Plane $Q_{z}$ Components of the Scattering Vector of the Mixed SP:DMPA = 1:1 Monolayer at $T=21^{\circ} \mathrm{C}^{a}$

\begin{tabular}{|c|c|c|c|c|c|c|c|}
\hline \multirow[b]{2}{*}{$\pi, \mathrm{mN} \mathrm{m}^{-1}$} & \multicolumn{3}{|c|}{ orthorrombic phase } & \multicolumn{4}{|c|}{ Overbeck phase } \\
\hline & $Q_{x y 1}, \AA^{-1}$ & $Q_{x y 2}, \AA^{-1}$ & $Q_{z}, \AA^{-1}$ & $Q_{x y 1}, \AA^{-1}$ & $Q_{x y 2}, \AA^{-1}$ & $Q_{z 1}, \AA^{-1}$ & $Q_{z 2}, \AA^{-1}$ \\
\hline 10 (directly after compression) & $1.446(0.112)$ & $1.582(0.033)$ & $0(0.34)$ & $1.453(0.053)$ & $1.526(0.080)$ & $0.231(0.23)$ & $0.462(0.23)$ \\
\hline 10 (after $40 \mathrm{~min})$ & $1.451(0.033)$ & $1.580(0.057$ & $0(0.35)$ & $1.469(0.031)$ & $1.521(0.052)$ & $0.23(0.22)$ & $0.46(0.22)$ \\
\hline 25 & $1.452(0.031)$ & $1.577(0.066)$ & $0(0.31)$ & $1.470(0.033)$ & $1.527(0.057)$ & $0.22(0.22)$ & $0.44(0.22)$ \\
\hline
\end{tabular}

${ }^{a}$ The full-width at half-maximum (fwhm) of the peaks are given in parentheses.

(Figure 3C), which are transformed into a lattice structure at high surface pressure (Figure 3D). As in the previous case, domains without structure are formed for $x=1 / 6$, but the domains coalesce in this case as the surface pressure increases. For high surface pressure, the plot of the area per molecule vs composition at a constant pressure shows a minimum at $x=$ 0.5 (see the Supporting Information), which agrees with the formation of a 1:1 complex. Moreover, only one collapse pressure is observed for all the binary mixtures, which is indicative of miscible components.

Additionally, some experiments for the SP:DMPA $=1: 1$ monolayer by using buffered subphase (acetic acid/acetate $10^{-5}$ M) were carried out. We observed that isotherms and BAM images do not show any differences from those obtained by using unbuffered subphase.

2. Synchrotron Grazing Incidence X-ray Diffraction (GIXD). Grazing Incidence X-ray Diffraction (GIXD) measurements have been performed in order to obtain quantitative information about the 2D symmetry of Langmuir monolayers. GIXD is sensitive only to the condensed parts of the monolayer, giving an ordered structure that can result in X-ray diffraction. The liquid expanded phase contributes to the background scattering. Structural changes along compression occurring for the SP:DMPA $=1: 1$ mixed monolayer have been monitored. The X-ray diffraction patterns were recorded before and after the overshoot of surface pressure (ca. $10 \mathrm{mN} / \mathrm{m}$ ). Moreover, the further effect of maintaining the surface pressure under a constant value of $10 \mathrm{mN} / \mathrm{m}$ has been studied, as it implies an elongation of the branches observed by BAM (see Figure 1). GIXD also has been performed at a higher value of surface pressure of $25 \mathrm{mN} / \mathrm{m}$. Figure 4 shows the corrected X-ray intensities as a function of the scattering vector components $Q_{x y}$ and $Q_{z}$ for the mixed SP:DMPA monolayer at $10 \mathrm{mN} / \mathrm{m}$ and different times after compression. Diffraction peaks and rods values are depicted in Table 1, among with their full-width at half-maximum values. Quantitative information on the unit cell parameters can be found in Table 2. Tilt angle and cross sectional area are also described. For comparison purposes, the pure DMPA monolayer has also been studied (see the Supporting Information). The pure DMPA monolayer shows three different phases, going from low to high surface pressures. At $5 \mathrm{mN} / \mathrm{m}$ the lipid forms an Overbeck (Ov) phase. With compression of the monolayer, at $10 \mathrm{mN} / \mathrm{m}$ the DMPA monolayer undergoes a phase transition to a $\mathrm{L}_{2}$ phase. In the $\mathrm{L}_{2}$ phase, the distortion of the lattice is significantly smaller. The tilt angle of the hydrocarbon chains is also reduced. At a high surface pressure of $30 \mathrm{mN} / \mathrm{m}$, the DMPA monolayer shows a LS phase, in which the lipid tails are untilted. The transition into the nontilted state occurs at $\sim 15 \mathrm{mN} / \mathrm{m}$. Therefore, an increase of the lipid ordering, as well as a progressive decrease of the in-plane area, is observed with compression of the monolayer (see the Supporting Information).

Figure 4A shows the diffraction patterns for the mixed monolayer SP:DMPA = 1:1 just before the overshoot of surface pressure (see isotherm in Figure 1), at ca. $10 \mathrm{mN} / \mathrm{m}$. Two crystalline phases can be distinguished: (a) an orthorhombic, 
TABLE 2: Primitive Unit Cell Parameters, Distortion Values, Tilt Angle, Projection of Hydrocarbon Chains in $x y$ Plane $\left(A_{x y}\right)$, and Cross Sectional Area of Hydrocarbon Chains $\left(A_{0}\right)$ of the Mixed SP:DMPA $=1: 1$ Monolayer at $T=21^{\circ} \mathrm{C}$

\begin{tabular}{|c|c|c|c|c|c|c|c|c|}
\hline$\pi, \mathrm{mN} \mathrm{m}^{-1}$ & $a, \AA$ & $b=c, \AA$ & $\alpha, \operatorname{deg}$ & $\beta=\gamma, \operatorname{deg}$ & distortion & tilt angle, deg & $A_{x y}, \AA^{2}$ & $A_{0}, \AA^{2}$ \\
\hline \multicolumn{9}{|c|}{ orthorhombic phase } \\
\hline 10 (directly after compression) & 4.465 & 4.485 & 125.6 & 117.2 & 0 & 0 & 19.4 & 19.4 \\
\hline 10 (after $20 \mathrm{~min}$ ) & 4.473 & 4.884 & 125.5 & 117.3 & 0 & 0 & 19.4 & 19.4 \\
\hline 25 & 4.488 & 4.874 & 125.2 & 117.4 & 0 & 0 & 19.4 & 19.4 \\
\hline \multicolumn{9}{|c|}{ Overbeck phase } \\
\hline 10 (directly after compression) & 5.081 & 4.838 & 116.6 & 121.7 & 0.0663 & 16.8 & 20.9 & 20.0 \\
\hline 25 & 5.002 & 4.815 & 117.4 & 121.3 & 0.0513 & 16.1 & 20.6 & 19.8 \\
\hline
\end{tabular}

untilted phase (this phase shows two Bragg peaks both at $Q_{z}=$ 0 , with $Q_{x y}=1.44 \AA^{-1}$ and $Q_{x y}=1.58 \AA^{-1}$; the hydrocarbon tails are tightly packed) and (b) an Overbeck (Ov) phase $\mathrm{p}^{47,48}$ showing two Bragg peaks at $Q_{x y}=1.45 \AA^{-1}, Q_{z}=0.23 \AA^{-1}$, and $Q_{x y}=1.52 \AA^{-1}, Q_{z}=0.46 \AA^{-1}$. The overshoot of surface pressure causes a modification of the mixed monolayer, observed by BAM as growth of branches (see Figure $1 \mathrm{C}-\mathrm{F}$ ). The effect of this mesoscopic rearrangement on the molecular structure of the mixed monolayer is described by using GIXD. Figure $4 \mathrm{~B}$ shows the diffraction patterns for the 1:1 mixed monolayer after a waiting time of ca. $30 \mathrm{~min}$. The Bragg peaks belonging to the orthorhombic phase give a weaker signal, indicating a decreasing amount with time in the mixed monolayer. However, they can still be quantified. The structural parameters of the orthorhombic phase do not significantly change with further compression of the mixed monolayer. Regarding the Ov phase, the intensity of the diffraction peaks increases their intensity with time at a constant pressure of $10 \mathrm{mN} / \mathrm{m}$. This increase indicates a larger contribution of this phase to the global structure of the monolayer. The distortion of the lattice decreases slightly with time, but the chain tilt and the cross-sectional area are unchanged (see Table 2). This relaxation may be related to the growth of the branches observed by BAM (Figure $1 \mathrm{C}-\mathrm{F}$ ). Figure 4C shows a decrease of the intensity for the orthorhombic phase Bragg peaks. An increase of the intensity for the Bragg peaks belonging to the Overbeck phase can also be observed. As seen in Table 2, there is no significant change in the structural parameters for the Overbeck and orthorhombic phases. The crystalline structure of the mixed monolayer has also been examined at a high value of surface pressure. Figure 4D shows the diffraction patterns for the 1:1 mixed monolayer at a surface pressure of $25 \mathrm{mN} / \mathrm{m}$. As noted previously, there is a further increase of intensity for Bragg peaks belonging to the Overbeck phase. This behavior permits us to relate the circular domains with the orthorrombic phase and the branches grown from the circles with the Overbeck phase.

As seen in Table 2, for the Overbeck phase a little decrease of the tilt angle of the hydrocarbon chains, while maintaining the packing, is observed. There is a slight increase in the distortion of the lattice. Additional information from the fullwidth at half-maximum of Bragg rods (fwhm $\left(Q_{z}\right)$ ) can be obtained. The length of the scatter $l$ can be defined as $l=$ $0.88(2 \pi) / \mathrm{fwhm}\left(Q_{z}\right){ }^{49}$ For the orthorhombic phase, $l$ is ca. 1.7 $\mathrm{nm}$, corresponding to the length of the DMPA hydrocarbon chains in the all-trans conformation. In the case of the Overbeck phase, $l$ is ca. $2.5 \mathrm{~nm}$. This length agrees well with the length of the hydrocarbon chain for the SP molecule in the all-trans conformation. Therefore, it is expected that in this phase the DMPA molecule is packed with the glycerol group region stretched and also forming part of the scattering unit. This conformation confirms the assumption in subsection 1 . On the basis of the shorter length of the DMPA chains, the DMPA molecule is able to interact to a higher extent, in this case even ordering the glycerol region, with SP molecules.

3. Reflection Spectroscopy and Determination of SP Polar Angle in the Mixed SP:DMPA Monolayers. Reflection spectroscopy detects only those molecules which are at the interface and contribute to enhanced reflection from the air-water interface. ${ }^{36}$ This technique gives us valuable information on the organization, density, and orientation of the chomophore molecules located at the air-water interface. ${ }^{3,19,41,44}$ Reflection spectra $\Delta R$ of SP:DMPA $=1: 1$ monolayers on the water subphase at different surface pressures are shown in Figure $5 \mathrm{~A}$.

For low values of absorption, the reflection $\Delta R$ has been shown to be proportional to the surface concentration of the dye, and is given in a reasonable approximation by ${ }^{16,19,36,41}$

$$
\Delta R=\left(2.303 \times 10^{3}\right) \Gamma f_{\text {orient }} \varepsilon \sqrt{R_{\mathrm{i}}}
$$

where $\Gamma$ is the surface concentration (in $\mathrm{mol} \mathrm{cm} \mathrm{cm}^{-2}$ ), $R_{\mathrm{i}}=0.02$, the reflectivity of the interface air-water at normal incidence,

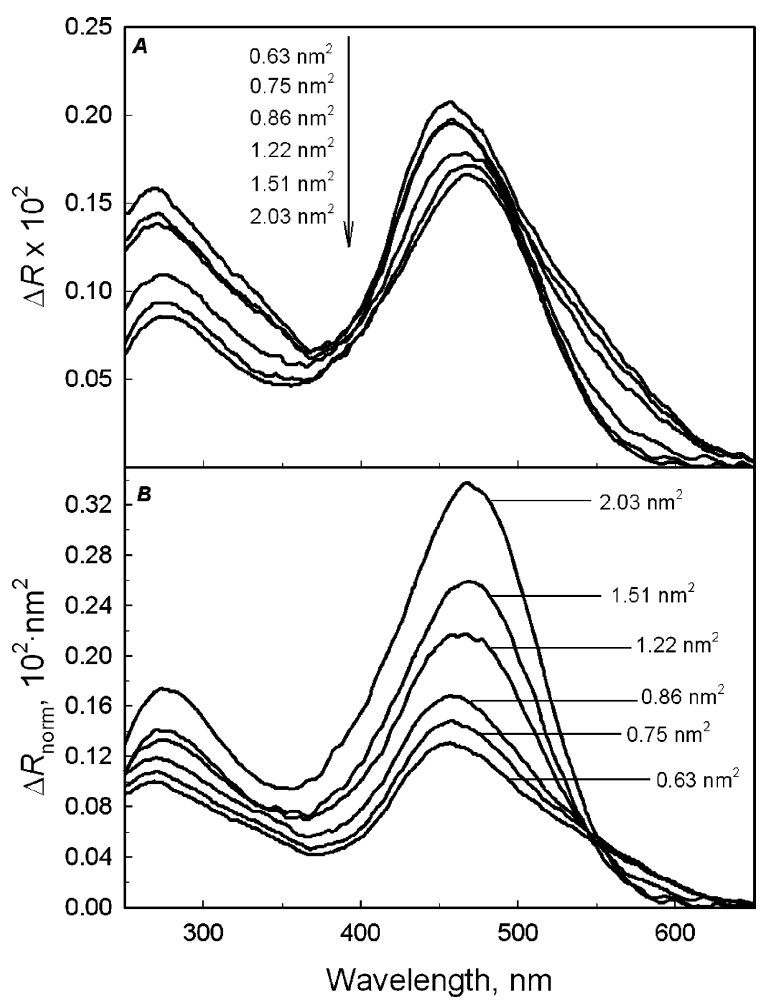

Figure 5. (A) Reflection spectra $\Delta R$ of the mixed SP:DMPA monolayers, molar ratio $1: 1$. The surface areas are indicated in the figure. (B) Normalized reflection spectra. 
$\varepsilon$ is the extinction coefficient (given as $\mathrm{L} \mathrm{mol}^{-1} \mathrm{~cm}^{-1}$ ), and $f_{\text {orient }}$ is a numerical factor that takes into account the different average orientation of the square transition moment of the dye in solution as compared to the monolayer at the air-water interface. For the domains observed in BAM images, the features of inner bright and dark regions indicate anisotropy. However, for a large region of the interface that includes numerous domains with a random distribution an isotropic film in the plane is considered and, therefore, there is not a preferential azimuthal angle, $\phi$. This just happens during the reflection measurements. In this case, $f_{\text {orient }}$ is ${ }^{19}$

$$
f_{\text {orient }}={ }^{3} / 2\left\langle\sin (\theta)^{2}\right\rangle
$$

where angle brackets indicate average values. The polar angle, $\theta$, is defined as the angle between the SP transition moment, $\mu$, and the normal to the air-water interface, while the azimuthal angle, $\phi$, is defined as the angle between the projection of $\mu$ on the $x-y$ plane and the $x$ axis (see Scheme 1B).

If we considered that $\Gamma=1 /\left(A \times N_{\mathrm{A}}\right)$, where $A$ is the area per SP molecule and $N_{\mathrm{A}}$ the Avogadro constant, we can rewrite eq 1 as

$$
\Delta R_{\text {norm }}=\Delta R \times A=\left(5.407 \times 10^{8}\right) f_{\text {orient }} \varepsilon
$$

The corresponding product $\Delta R \times A=\Delta R_{\text {norm }}$ is shown in Figure $5 \mathrm{~B}$. The quantity $\Delta R_{\text {norm }}$ is proportional to the product $f_{\text {orient }} \varepsilon$, and represents the reflection normalized to the surface density of SP. Therefore, they show more clearly the changes of orientation and/or association than the directly measured spectra.

At low surface pressures $(\pi<5 \mathrm{mN} / \mathrm{m})$, the spectra present a low-energy band at $475 \mathrm{~nm}$, corresponding to the $\pi-\pi^{*}$ transition over the chromophore in the trans configuration, and a band at $265 \mathrm{~nm}$ arising from the isolated transition on the pyridinium and phenyl moieties. ${ }^{50,51}$ As the surface pressure increases, some phenomena take place in the reflection band: (1) $\Delta R_{\text {norm }}$ at 475 and $265 \mathrm{~nm}$ decreases, which should be related to the decreasing of the polar tilt angle of the chromophores and (2) for $\pi>5 \mathrm{mN} / \mathrm{m}$, the maximum wavelength of the lowenergy band shifts to shorter wavelengths (from 475 to $459 \mathrm{~nm}$ ), this fact must be attributed to the formation of $\mathrm{H}$ aggregates of the SP chromophores. ${ }^{37,50-52}$

The oscillator strength is defined as ${ }^{53}$

$$
f=\frac{4 \varepsilon_{0} 2.303 m_{\mathrm{e}} c_{0}}{N_{\mathrm{A}} e^{2}} \int_{\mathrm{Band}} \varepsilon \mathrm{d} v=1.44 \times 10^{-19} \int_{\mathrm{Band}} \varepsilon \mathrm{d} v
$$

where $\varepsilon_{0}$ is the permittivity of the vacuum, $m_{\mathrm{e}}$ is the electron mass, $e$ is the elementary charge, and $c_{0}$ is the speed of light in a vacuum. In eq 4, the numerical factor $1.44 \times 10^{-19}$ is expressed in mol L $\mathrm{L}^{-1} \mathrm{~cm} \mathrm{~s}$. From eqs 1,3 , and 4 , it is possible to define an apparent oscillator strength determined from the measured reflection spectra as ${ }^{19}$

$$
f_{\text {app }}=f \times f_{\text {orient }}=2.6 \times 10^{-12} \int_{\text {Band }} \Delta R_{\text {norm }} \mathrm{d} v
$$

where the numeric factor $2.6 \times 10^{-12}$ is expressed in $\mathrm{nm}^{-2} \mathrm{~s}$. Moreover, the ratio between the apparent oscillator transition and the oscillator strength from the solution gives the orientation factor, $f_{\text {orient }}=f_{\text {app }} / f$. If the oscillator strength for any aggregation degree is conserved, according to the Davidov model, ${ }^{8,53-55}$ the average polar tilt angle $\theta$ could be evaluated by using eq 2 . However, the oscillator strength of the hemicyanine group depends on the solvent polarity, ${ }^{13}$ so we do not have a proper reference value for $f$.

An alternative way to estimate the chromophore angle is assuming that the hemicyanine group is located flat with respect to the interface for large surface areas, that is, $\theta \approx 90^{\circ}$. In these cases, for $A>1.8 \mathrm{~nm}^{2}, f_{\text {app }} \approx 1.2$ has been calculated, and thus the oscillator strength is $f \approx 0.8$ (eqs 2 and 5). This value is close to those published for species with similar dye groups, and ranged between 0.67 and $0.78 .{ }^{13}$

The interface is completely covered by domains for $A \leq 0.7$ $\mathrm{nm}^{2}$, where the surface pressure increases sharply (see Figure 1). $A \approx 0.7 \mathrm{~nm}^{2}$ should be the area corresponding to that occupied for hemicyanine molecule (or per DMPA) in the circular and branch domains. For these areas, values of $f_{\text {app }} \approx$ 0.7 were obtained, and according to eq $2, \theta \approx 50^{\circ}$.

Therefore, the reflection spectra analysis shows two fundamental phenomena as the surface pressure increases, first the formation of $\mathrm{H}$ aggregates (shifting of the visible band toward lower wavelengths), and second the tilt of the hemicyanine group (decreasing of the apparent oscillator strength).

The area limit per SP molecule in the pure dye film is close to $A_{0} \approx 0.33 \mathrm{~nm}^{2},{ }^{13}$ and corresponds to a vertical orientation of the hemicyanine group. The polar angle obtained, $\theta \approx 50^{\circ}$, is indicative that the hemicyanine group extends to occupy much of the available area at the air-water interface. The projected area of the chromophore onto the surface assuming a tilt angle of $50^{\circ}$ has been estimated by means of HypeChem $6.0,{ }^{56}$ obtaining a value of $0.64 \mathrm{~nm}^{2}$, approximately. This value agrees with the idea that the polar group of DMPA hardly occupies a net area at the interface, and is probably located next to the hemicyanine pyridyl group, but immersed in the boundary between hydrophobic and hydrophilic regions (see Scheme 1B).

4. The Organization of Hemicyanine Polar Group and the Simulation of Circles Domain Textures. Scheme 2A shows some circular domains observed by BAM at different temperatures. Also, Scheme 2B shows some domains observed at $17{ }^{\circ} \mathrm{C}$ for a second cycle under compression. As discussed earlier, the texture of these domains is the same, that is, with bright lateral and dark vertical regions.

The experimental BAM reflectivity is a relative amount. If the exposition time to the reflected light (auto set function in the BAM equipment) is increased, improved resolution of the dark regions is achieved, countering the decreased resolution for the bright areas (Scheme 2C). In this case, although the general texture is similar to that observed previously at $21^{\circ} \mathrm{C}$, the circular domain textures show a thin vertical gray region surrounded by darker regions (see Scheme 2C).

In a BAM experiment, p-polarized light reaches the air-water interface with an angle $\alpha=53.15^{\circ}$, Brewster angle. Usually, the different BAM textures are observed due to changes of the refractive index resulting from differences in thickness, density, and/or molecular orientation between the different regions of the film. However, for our system, the SP molecules absorb at the $532 \mathrm{~nm}$ laser beam (see Figure 3). As previously suggested, the domain formation is induced by the SP aggregation whose absorption originates the strong changes in the film refractivity. Therefore, this phenomenon would be mainly responsible for the appearance in a first approximation of domain textures. In such a case, the Fresnel equations for biaxial 
SCHEME 2: (A) Zoom of Experimental Circular Domains Observed by BAM at Different Temperatures; (B) Domains Observed at $17^{\circ} \mathrm{C}$ during the Second Cycle of Compression; (C) Domains Observed at $17{ }^{\circ} \mathrm{C}$ during the Second Cycle of Compression by Increasing the Opening Time of the BAM Camera Lens; (D) Organization Model of the 2D Circular Domain of the Mixed SP:DMPA $=1: 1$ Film and Definition of $\gamma$ (the angle formed between the $x$ axis and any radial circle), and $\beta$ (angle between projection of the dipole moments on the $x-y$ plane and the radial direction); ${ }^{a}(\mathrm{E})$ Concentric Configuration $\left(\beta=90^{\circ}\right)$; and (F) Radial Configuration $\left(\beta=0^{\circ}\right)$

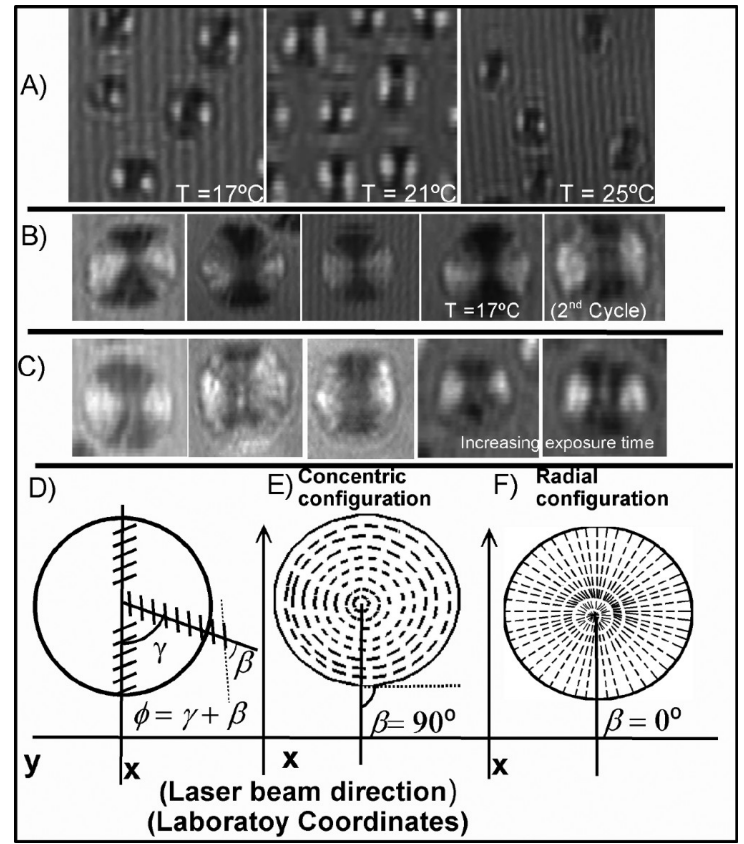

${ }^{a}$ Dashed lines represent the projection of the transition dipole on the $x-y$ plane.

anisotropic materials on isotropic substrate should be used to calculate the reflection of the film. ${ }^{57}$

The SP:DMPA $=1: 1$ mixed film is assumed to be a biaxial material with complex refractive indices of $N_{q}=n_{q}-i k_{q}(q \equiv$ $x, y$, and $z$, see the Supporting Information).

To obtain the BAM images the analyzer was fixed at $0^{\circ}$, and therefore, only the p-component of the reflection is detected. Thus, the $N_{y}$ component does not affect the final result. Moreover, the absorption coefficients can be expressed as follows: $k_{x}=k_{0} \times \sin (\theta) \times \cos (\phi)$, and $k_{z}=k_{0} \times \cos (\theta)$. Assuming values for the different constants $\left(k_{0}, n_{x}, n_{z}, \theta, \phi\right.$, and the thickness of the film, $d$ ), the absolute reflectivity of the film can be determined. Following, this absolute reflection must be transformed into a relative gray level scale (from 0 to 255) for the simulation of the domain texture. Details of the simulation procedure are given in the Supporting Information. From reflection spectra $\theta \approx 50^{\circ}$ is calculated, being estimated from the values $k_{0} \approx 0.15, n_{x}=n_{z} \approx 1.6$, and $d \approx 3.7 \mathrm{~nm}$. In such a way, the reflectivity depends exclusively on $\phi$. This simulation model is only approximate because the anisotropy of the DMPA alkyl chains is not considered.

From now on, the axis $x$ (laboratory coordinates) is defined as the projection of the laser incidence axis on the interface (see Scheme 2D).

If the presence of the vertical gray region in the circular domains is ignored, at this discussion point, we can consider

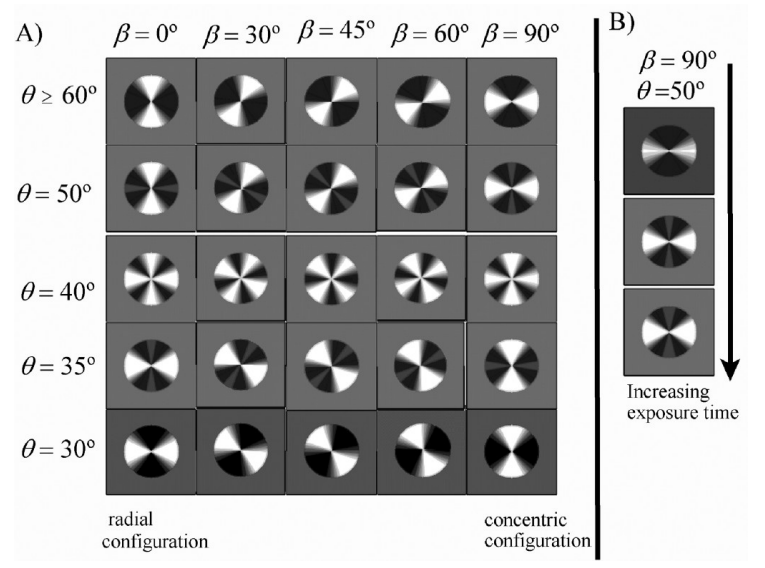

Figure 6. (A) Circular domain simulation for different polar angle tilt, $\theta$, and different angle $\beta$, by using $k_{0}=0.15, n_{x}=n_{z}=1.6$, and $d$ $=3.7 \mathrm{~nm}$. (B) Theoretical texture of the circular domains, for $\theta=51^{\circ}$ and $\beta=90^{\circ}$, where the exposure time to the light is simulated (see the Supporting Information).

that the reflectivity inside each circle changes progressively with the rotation angle, being brighter at the lateral position of the circle than at the vertical position. Also, the reflectivity is almost constant for any radius of the circle, which permits us to suppose that the azimuthal angle $\phi$ of the hemicyanine group is constant for a given radial direction (see Scheme 2D). In any case, some small changes in the texture can be appreciated, which should be linked to fluctuations in the molecular orientation.

The angle formed between the $x$ axis and any radius of the circle (circle rotation angle) is denoted by $\gamma$, and $\beta$ is the angle between the projection of the dipoles moment on the $x-y$ plane and the radial direction (see Scheme 2D). In this way, the azimuthal angle is $\phi=\gamma+\beta$. Parts $\mathrm{E}$ and $\mathrm{F}$ of Scheme 2 show sketches for $\beta=90^{\circ}$ (concentric configuration) and $\beta=0^{\circ}$ (radial configuration), respectively, where dashed lines represent the projection of the transition dipole on the $x-y$ plane.

As previously observed, the regions located at the lateral position of the experimental circle domains are always brighter than those located at the vertical position. Usually, this type of texture indicates a preferential perpendicular orientation of the transition dipoles along the radial direction $\left(\beta=90^{\circ}\right.$, Scheme $2 \mathrm{E}$, concentric configuration). However, the reflected light ( $\mathrm{p}$ polarized) of thin films under Brewster angle incidence is not a linear phenomenon and the simulation is necessary to clarify some experimental details as, for example, the thin gray vertical region observed in the texture domain. As discussed below, this phenomenon is not due to a change in the dipole orientation, but an optical effect resulting from the reflection at the Brewster angle incidence.

Figure 6A shows the results of the circular domain simulation for different polar tilt angles $\theta$, and for different $\beta$ angles, using $k_{0}, n_{x}, n_{z}$, and $d$ values estimated previously. In any case, the simulated brightness (reflectivity) of the domains is a relative amount, and is related to the minimum and maximum reflection of each case (see the Supporting Information). We have observed that for $\theta \geq 60^{\circ}$ and $\beta=90^{\circ}$ (concentric configuration), the texture appearance of the domain is approximately coincident with that experimentally registered (parts A and B in Scheme 2 ). However, for $\theta \leq 30^{\circ}$, where the absolute reflectivity is low (see the Supporting Information), the texture appearance is also coincident with the experimental domains when $\beta=0^{\circ}$ (radial configuration) (Scheme 2A,B).

For $\theta=50^{\circ}$ and $\beta=90^{\circ}$, and for $\theta=35^{\circ}$ and $\beta=0^{\circ}$, the model predicts the appearance of a narrow gray region along 


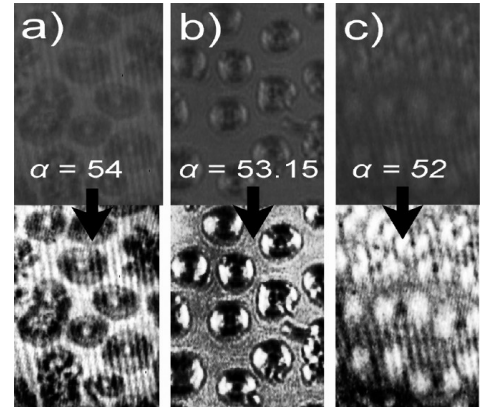

Figure 7. Top: Experimental domains texture $\left(T=17^{\circ} \mathrm{C}\right)$ by using $\alpha=52^{\circ}, 53.15^{\circ}$, and $54^{\circ}$. Bottom: Equalized images.

the vertical direction of the circular domain, surrounded by darker regions (see Figure 6A). This fact agrees with the experimental texture observed when the reflectivity increases (see Scheme 2C). The theoretical textures of the circular domains, for $\theta=50^{\circ}$ (from reflection spectroscopic analysis) and $\beta=90^{\circ}$, are shown in Figure 6B, where the exposure time to the light also has been simulated (see the Supporting Information). The results show the thin gray vertical region could be better defined as the exposure time increases. For $\beta=$ $90^{\circ}$, the size of this gray region decreases as $\theta$ increases, disappearing completely when $\theta \geq 60^{\circ}$. For $\beta=0^{\circ}$, the size of this gray region decreases as $\theta$ decreases, disappearing completely for $\theta=30^{\circ}$.

Unfortunately, the theoretical texture changes as a function of $\theta$ (Figure 6A) cannot be observed because the experimental polar angle remains almost constant under any experimental condition, even when the temperature is changed. Therefore, the two situations in which the texture of the simulated domains matches the experimental texture, that is, $\theta \approx 50^{\circ}$ and $\beta=90^{\circ}$, or $\theta \approx 35^{\circ}$ and $\beta=0^{\circ}$, cannot be distinguished.

However, it is possible to change the texture of the experimental domains varying slightly the incidence angle of the polarized light. Thus, for the $k_{0}, n_{y}, n_{z}$, and $d$ values used previously, the Fresnel's equations predict the maximum reflection for $\phi=0^{\circ}$ and $180^{\circ}$ when $\alpha \leq 53^{\circ}$, and independently of the $\theta$ value, whereas if $\alpha \geq 53.8^{\circ}$, the reflection is maximum for $\phi=90^{\circ}$ and $270^{\circ}$, also for any $\theta$ value (see the Supporting Information). The top portions of panels $\mathrm{a}, \mathrm{b}$, and $\mathrm{c}$ of Figure 7 show domain textures with $\alpha=52^{\circ}, 53.15^{\circ}$, and $54^{\circ}$, respectively.

For $\alpha=52^{\circ}$ and $54^{\circ}$, the reflectivity increases considerably, and image resolution becomes worse. To improve such a situation, the images in Figure 7 were equalized (bottom). As is clearly observed, the domain texture is inverted when $\alpha$ changes from $52^{\circ}$ to $54^{\circ}$. The key to distinguish between the two options proposed by the simulation $\left(\theta \approx 50^{\circ}\right.$ and $\beta=90^{\circ}$, or $\theta \approx 35^{\circ}$ and $\beta=0^{\circ}$ ) is the following: for the experimental domain, the texture is the same for $\alpha=52^{\circ}$ and $53.15^{\circ}$, and therefore, in both cases the maximum reflectivity corresponds to $\phi=0^{\circ}$ and $180^{\circ}$. In these cases, since the bright regions are located in the lateral positions of the circles, $\gamma=90^{\circ}$ or $270^{\circ}$, and with $\phi=\gamma+\beta, \beta=90^{\circ}$ or $270^{\circ}$ can be deduced, that is, a concentric configuration. Therefore, one could argue that $\theta$ $\approx 50^{\circ}$. This result is coincident with that previously obtained from the reflection spectra.

Inside the circular domains obtained for the SP:DMPA = 1:1 system, the hemicyanine group adopts the concentric configuration but, in any case, the alkyl chains rearrange in orthorhombic configuration, as tested by GIXD.
SCHEME 3: (A) Circular Domains Observed at $17^{\circ} \mathrm{C}$ (second compression process); (B) Model of the Branch Growth, and Definition of Director, n; (C) Simultaneous Changes of Reflectivity and the Direction of Growth for Branch Domains, and a Sketch Interpreting Such Changes As a Function of the Direction Changes of the Director, n; (D) Drawing of the Aggregation of the Hemicyanine Group; and (E) Model of Fan-Type Configuration for Branch Domains

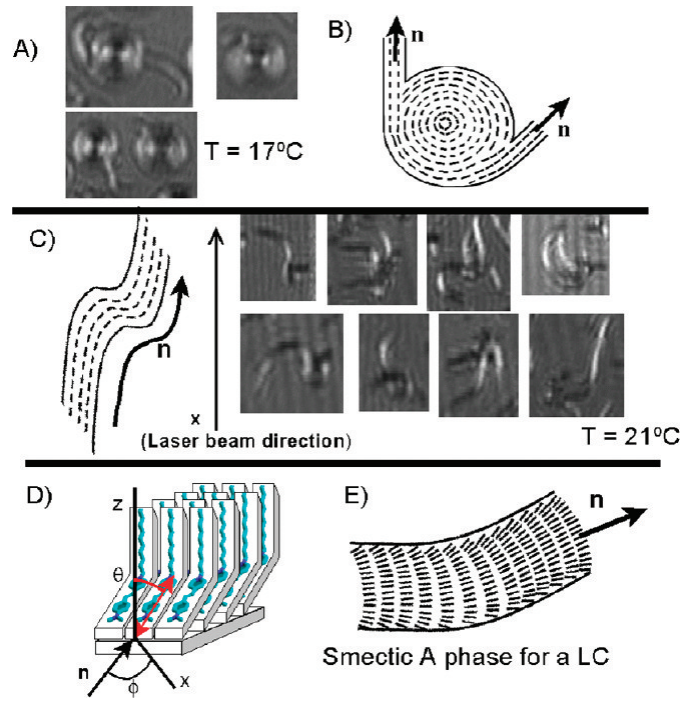

5. Branches Formation and Hemicyanine Polar Group Organization. At $17^{\circ} \mathrm{C}$, during the second compression process, and when the circular domains are large enough, rings are formed around them, which are broken away from the central structure. This phenomenon is clearly observed in Figure 2G and Scheme 3A, and may help us to understand the mechanism of formation and growth of branches arising from the circular domains.

In circular domains, the SP molecules are aggregated along the radial direction with $\beta=90^{\circ}$ (concentric configuration, see Scheme 2D), which somehow must minimize the domain energy. However, this energetic advantage seems to disappear when the domain grows too much, at least for external molecules whose circle curvature becomes negligible. Likewise, neither circular domains nor branches growing from them coalesce under compression, indicating an anisotropic line tension. ${ }^{58-60}$ The anisotropic line tension may be originated by the different tilt of the lipid at the boundary of the domains, similar to the behavior of the liquid crystal. ${ }^{61}$ In this case, as in liquid crystals, small defects originate the formation of regions or branches that grow independently of the central domain, and for which anisotropic line tension prevents their coalescence with the central domain. The number of defects increases with the temperature, and so does the proliferation of the number of branches arising from circular domains.

Once a branch is formed after the breakup of a ring segment of the circular domain, its growth is only along and not across the branch. Therefore, the growth of this branch-like domain is different from that for circular domains. As for liquid crystal phases, the average projection of the dipole transition in the interface could be described by a unit vector called the director and denoted by $\mathbf{n}$ (see Scheme 3B). Because of the branch growth, the molecular aggregation is the phenomenon that defines the $\mathbf{n}$ direction. The molecular aggregation at the end of the branches is energetically favorable with respect to the aggregation at the circular domains. As commented on previ- 
ously, the brightness of these branches depends on the direction of their growth, that is, of the $\mathbf{n}$ direction. Thus, branches grown along the vertical direction are bright, while they are dark in the horizontal direction. Intermediate brightness is obtained as a function of the angle between the $x$ axis and $\mathbf{n}$. When a branch changes its direction growth, its brightness changes simultaneously (see Scheme 3C). These changes in the brightness of the branches should be interpreted due to changes in the direction of the molecular aggregation, because of fluctuations or collisions with other domains. In any case, we must remember that BAM reflectivity is a relative magnitude.

A sketch of the aggregation of the hemicyanine group is depicted in Scheme 3D. For simplicity, the DMPA molecule is not represented. The model resembles two-dimensional smectic A phase (see Scheme 3E), similar to that described for liquid crystal where there are positional order along the director and the layer normal to the director. In this case, the azimuthal angle, $\phi$, is defined by the angle between $\mathbf{n}$ and the $x$ axis (laboratory coordinates). Anyhow, we must remember that the alkyl chains are packed in an Overbeck phase, as by GIXD. However, for the polar group, the orientation order is not perfect. For example, Scheme 3C shows how for some branches growing in the vertical direction, the central region of the branch is brighter than the lateral regions, which is related to the tilt of the dipole with respect to the director, possibly in a fan-type configuration (see Scheme 3E). This phenomenon increases with the temperature (see branched domains in Figure 2C) and can be related to the anisotropic line tension of the domains.

\section{Conclusions}

In this work, the formation of mesoscopic 2D structures at the air-water interface from mixed SP:DMPA monolayers has been reported. For this system, lateral aggregation of the hemicyanine group induces the formation of domains with longrange order, showing different textures and indicating the existence of anisotropy. Thus, circular domains with bright horizontal regions and dark vertical regions have been observed. Furthermore, depending on the temperature, it is observed how branches grow from circular domains, whose brightness depends on the growth direction. Thus, BAM images allow us to see some branches that change their growth direction and their brightness simultaneously.

GIXD experiments for the SP:DMPA $=1: 1$ mixed monolayer show the coexistence of two phases, orthorhombic and Overbeck, at $10 \mathrm{mN} / \mathrm{m}$, which account for the packaging of the alkyl chains. With increasing the waiting time at $10 \mathrm{mN} / \mathrm{m}$, the Overbeck phase is increased at the expense of the orthorhombic phase, which increases its contribution to the global structure along the isotherm. At a high value of surface pressure of 25 $\mathrm{mN} / \mathrm{m}$, almost all the mixed monolayer is in the Overbeck phase, although there is still a small part of the monolayer in the orthorhombic phase. The orthorhombic phase structure is not modified along the isotherm, while the Overbeck phase structure is slightly modified under compression. This behavior permits us to relate the circular domains with the orthorhombic phase and the branches grown from the circles with the Overbeck phase. However, the texture of the domain formed and observed by BAM does not depend on the packing of alkyl chains, but the rearrangement of the hemicyanine polar group, because this group absorbs at the laser wavelength used in BAM.

The experimental circular domain textures have been simulated on the basis of the Fresnel equations for biaxial anisotropic materials on isotropic substrates. Therefore, the refractivity of the film can be calculated. In our simulation model, it has been assumed that the reflectivity is only caused by the absorption of the hemicyanine group. The simulation results support that the tilt angle of the hemicyanine group must be around $50^{\circ}$ (polar angle), in good agreement with the value obtained from reflection spectra at the air-water interface. The light reflection (p-polarized) of thin films under Brewster angle incidence is a complex phenomenon and the simulation is necessary to clarify some experimental details as, for example, the thin gray vertical region surrounded by darker regions in the texture of circular domains. As demonstrated, this phenomenon is not due to a change in the dipole orientation, but to an optical phenomenon observed for certain values of polar angle, when the reflectivity is measured at the Brewster angle.

The experimental data obtained by means of GIXD and BAM are complementary and give us information about the rearrangement in the lipid (GIXD) and polar (BAM) regions of the monolayer, respectively. It is the hemicyanine aggregation that induces the domain formation, but there must be geometrical compatibility between the polar groups and alkyl chains to make possible the formation of large domains with well-defined structures.

The geometry of the structures formed depends crucially on a proper balance between the vertical sections of the hydrophobic and hydrophilic groups. We design $a_{\mathrm{c}}$ as the interfacial area occupied by alkyl chains when they are fully extended, i.e. at high surface pressures. Thus, for the SP:DMPA $=1: 1$ monolayer, $a_{\mathrm{c}} \approx 0.6 \mathrm{~nm}^{2}$. Moreover, we design $a_{0}$ as the minimum interfacial area occupied by the surfactant hydrophilic or headgroup. Monolayers with $a_{0}>a_{\mathrm{c}}$ could result in stable but heterogeneous films, as for SP monolayers without DMPA (see the Supporting Information), where $a_{\mathrm{c}} \approx 0.2 \mathrm{~nm}^{2}$ and $a_{0}$ $\approx 0.33 \mathrm{~nm}^{2}$. When $a_{0} \ll a_{\mathrm{c}}$, homogeneous monolayers can be obtained, although generally unstable, and consequently with low collapse pressures. This happens, for example, for monolayers formed by substances without polar group, such as semifluorinated alkanes. ${ }^{62,63}$ In general for homogeneous, insoluble, and stable monolayers, $a_{0} \leq a_{\mathrm{c}}$, although the interactions between hydrophilic groups determine to a great extent the properties of the monolayer. For the mixed SP:DMPA $=1: 1$ monolayer, we cannot determine the contribution of the DMPA polar group to the $a_{0}$ value. However, the experimental results indicate that the hemicyanine group tilts to fill the available surface excess at the interface $\left(a_{\mathrm{c}}-a_{0}\right)$, resulting in a effective packing where $a_{0} \approx a_{\mathrm{c}}$. The high lateral aggregation energy of the hemicyanine group counters the repulsion between dipole moments in the monolayer. This fact stabilizes the monolayer and enables the domains growth to mesoscopic sizes. In this way, the domain structure depends on the ability of the dye to fill the available area.

Acknowledgment. The authors thank the Spanish CICYT for financial support of this research in the framework of Project CTQ2007-64474/BQU (FEDER A), and also thank the Junta de Andalucía (Consejería de Innovación, Ciencia y Empresa) for special financial support (P06-FQM-01698 and P08-FQM4011). A. González-Delgado, C. Roldán-Carmona, and J. J. Giner-Casares thank the Ministerio de Ciencia e Innovación for their corresponding predoctoral grants (Formación de Profesorado Universitario, FPU) and the Alexander von Humboldt Foundation for a postdoctoral fellowship.

Supporting Information Available: Surface pressure-area isotherms and Brewster angle microscopy of pure SP, area per molecule vs mole fraction of SP at different surface pressures, GIXD of pure DMPA monolayer, reflection of an anisotropic 
thin film on isotropic substrate and simulation of circles domain textures, and reflection of an anisotropic thin film on isotropic substrate, under several incidence angles. This material is available free of charge via the Internet at http://pubs.acs.org.

\section{References and Notes}

(1) Lehn, J.-M. Angew. Chem., Int. Ed. Engl. 1990, 29, 1304. 17635.

(2) Vollhardt, D.; Liu, F.; Rudert, R. J. Phys. Chem. B 2005, 109,

(3) Pérez-Morales, M.; Pedrosa, J. M.; Martín-Romero, M. T.; Möbius, D.; Camacho, L. J. Phys. Chem. B 2004, 108, 4457.

(4) Whitesides, G. M.; Grzybowski, B. Science 2002, 295, 2418.

(5) Kaganer, V. M.; Möhwald, H.; Dutta, P. Rev. Mod. Phys. 1999, $71,779$.

(6) Thirumoorthy, K.; Nandi, N.; Vollhardt, D. J. Phys. Chem. B 2005, 109, 10820 .

(7) Johann, R.; Vollhardt, D.; Möhwald, H. Colloid Polym. Sci. 2000, $278,104$.

(8) Giner-Casares, J. J.; de Miguel, G.; Pérez-Morales, M.; MartínRomero, M. T.; Muñoz, E.; Camacho, L. J. Phys. Chem. C 2009, 113, 5711.

(9) Young, M. C. J.; Jones, R.; Tredgold, R. H.; Lu, W. X.; Ali-Adib, Z.; Hodge, P.; Abbasi, F. Thin Solid Films 1989, 182, 319.

(10) Kajikawa, K.; Anzai, T.; Shirota, K.; Takezoe, H.; Fukuda, A. Thin Solid Films 1992, 210, 699.

(11) Schildkraut, J. S.; Penner, T. L.; Willand, C. S.; Ulman, A. Opt. Lett. 1988, 13, 134.

(12) Carpenter, M. A.; Willand, C. S.; Penner, T. L.; Williams, D. J.; Mukamelt, S. J. Phys. Chem. 1992, 96, 2801.

(13) Turshatov, A. A.; Mobius, D.; Bossi, M. L.; Hell, S. W.; Vedernikov, A. I.; Lobova, N. A.; Gromov, S. P.; Alfimov, M. V.; Zaitsev,

S. Y. Langmuir 2006, 22, 1571 .

(14) Turshatov, A. A.; Zaitsev, S. Y.; Sazonov, S. K.; Vedernikov, A. I.; Gromov, S. P.; Alfimovc, M. V.; Möbius, D. Colloids Surf., A 2008, 329, 18.

(15) Fang, J. Y.; Uphaus, R. A.; Stroeve, P. Thin Solid Films 1994, 243,450

(16) Orrit, M.; Möbius, D.; Lehmann, U.; Meyer, H. J. Chem. Phys. 1986, 85, 4966.

(17) Kawai, T.; Umemura, J.; Takenaka, T. Langmuir 1989, 5, 1378.

(18) Ahuja, R. C.; Möbius, D. Langmuir 1992, 8, 1136.

(19) Pedrosa, J. M.; Martín-Romero, M. T.; Camacho, L.; Möbius, D.

J. Phys. Chem. B 2002, 106, 2583.

(20) Hönig, D.; Möbius, D. J. Phys. Chem. 1991, 95, 4590.

(21) Hönig, D.; Overbeck, G. A.; Möbius, D. Adv. Mater. 1992, 4, 419.

(22) Johann, R.; Vollhardt, D. Mater. Sci. Eng., C 1999, 8-9, 35.

(23) Krasteva, N.; Vollhardt, D. Colloids Surf., A 2000, 171, 49.

(24) Weidemann, G.; Gehlert, U.; Vollhardt, D. Langmuir 1995, 11, 864.

(25) Vollhardt, D.; Melzer, V. J. Phys. Chem. 1997, 101, 3370.

(26) Kaganer, V. M.; Peterson, I. R.; Kenn, R. M.; Shih, M. C.; Durbin, M.; Dutta, P. J. Chem. Phys. 1995, 102, 9412.

(27) Jacquemain, D.; Leveiller, F.; Weisbuch, S.; Lahav, M.; Leiserowitz,

L.; Kjaer, K.; Als-Nielsen, J. J. Am. Chem. Soc. 1991, 113, 7684.

(28) Als-Nielsen, J.; Jaquemain, D.; Kjaer, K.; Lahav, M.; Levellier,

F.; Leiserowitz, L. Phys. Rep. 1994, 246, 251.

(29) Kjaer, K. Phys. B 1994, 198, 100.
(30) Brezesinski, G.; Dietrich, A.; Struth, B.; Böhm, C.; Bouwman, W. G.; Kjaer, K.; Möhwald, H. Chem. Phys. Lipids 1995, 76, 145.

(31) Overbeck, G. A.; Hönig, D.; Möbius, D. Thin Solid Films 1994, 242,213

(32) Weidemann, G.; Vollhardt, D. Langmuir 1996, 12, 5114.

(33) Weidemann, G.; Brezesinski, G.; Vollhardt, D.; Möhwald, H. J. Phys. Chem. B 1998, 102, 1224.

(34) Ignés-Mullol, J.; Claret, J.; Reigada, R.; Sagués, F. Phys. Rep. 2007, $448,163$.

(35) Nandi, N.; Vollhardt, D. Chem. Rev. 2003, 103, 4033.

(36) Grüniger, H.; Möbius, D.; Meyer, H. J. Chem. Phys. 1983, 79, 3701.

(37) Hall, R. A.; Thistlethwaite, P. J.; Grieser, F.; Kimizuka, N.; Kunitake, T. Colloids Surf., A 1995, 103, 167.

(38) Vollhardt, D.; Fainerman, V. B. J. Phys. Chem. B 2002, 106, 345.

(39) Lösche, M.; Duwe, H. P.; Möhwald, H. J. Colloid Interface Sci.

1988, 126, 432.

(40) Pérez-Morales, M.; Pedrosa, J. M.; Muñoz, E.; Martín-Romero,

M. T.; Möbius, D.; Camacho, L. Thin Solid Films 2005, 488, 247.

(41) Martin, M. T.; Prieto, I.; Camacho, L.; Möbius, D. Langmuir 1996, 12,6554 .

(42) Giner-Casares, J. J.; Perez-Morales, M.; Bolink, H.; Muñoz, E.; de Miguel, G.; Martin-Romero, M. T.; Camacho, L. J. Colloid Interface Sci. 2007, 315, 278.

(43) Prieto, I.; Martín-Romero, M. T.; Camacho, L.; Möbius, D. Langmuir 1998, 14, 4175.

(44) Ahuja, R. C.; Caruso, P. L.; Möbius, D.; Wildburg, G.; Ringsdorf, H.; Philp, D.; Preece, J. A.; Stoddart, J. F. Langmuir 1993, 9, 1534.

(45) Giner-Casares, J. J.; Perez-Morales, M.; Bolink, H. J.; Munoz, E.; de Miguel, G.; Martin-Romero, M. T.; Camacho, L. J. Colloid Interface Sci. 2007, 315, 278

(46) Prieto, I.; Martín-Romero, M. T.; Camacho, L.; Mobius, D. Langmuir 1998, 14, 4175.

(47) Overbeck, G. A.; Möbius, D. J. Phys. Chem. 1993, 97, 7999.

(48) Overbeck, G. A.; Hönig, D.; Möbius, D. Langmuir 1993, 9, 555.

(49) Leveiller, F.; Jacquemain, D.; Leiserowitz, L.; Kjaer, K.; AlsNielsen, J. J. Phys. Chem. 1992, 96, 10380.

(50) Song, Q.; Evans, C. E.; Bohn, P. W. J. Phys. Chem. 1993, 97, 13736.

(51) Lusk, A. L.; Bohn, P. W. Langmuir 2000, 16, 9131.

(52) Lusk, A. L.; Bohn, P. W. J. Phys. Chem. B 2001, 105, 462.

(53) Kuhn, H.; Försterling, H. D. Principles of Physical Chemistry; Jonh Wiley \& Sons: New York, 1999.

(54) Kobayashi, T. J-Aggregates; World Scientific: Singapore, 1996.

(55) Pope, M.; Swenberg, C. E. Electronic Processes in Organic Crystals and Polymers; Oxford Science Publications: New York, 1999.

(56) Hyperchem, 6th ed.; Hypercube, Inc.: Gainesville, FL, 1999

(57) Fujiwara, H. Spectroscopic Ellipsometry. Principles and Applications; John Wiley \& Sons: Chichester, England, 2007.

(58) Henon, S.; Meunier, J. Chem. Phys. 1993, 98, 9148.

(59) Riviére, S.; Meunier, J. Phys. Rev. Lett. 1995, 74, 2495.

(60) Mufazzal-Hossain, M.; Kato, T. Langmuir 2000, 16, 10175.

(61) Yamamoto, T.; Manaka, T.; Iwamoto, M. Eur. Phys. J. E: Soft Matter Biol. Phys. 2009, 29, 1.

(62) Gaines, G. L. Langmuir 1991, 7, 3054.

(63) Dynarowicz-Latka, P.; Pérez-Morales, M.; Muñoz, E.; Broniatowski, M.; Martín-Romero, M. T.; Camacho, L. J. Phys. Chem. B 2006, 110, 6095.

JP1076577 